\title{
Impact of the Association of a High Fructose Diet and Chronic Mild Stress on Metabolic and Affective Disorders in Male Rat
}

\author{
Y. Chahirou*, M. Lamtai, A. Mesfioui, A. Ouichou, M. Coulibaly, R. Boussekkour, A. El Hessni \\ Unit of Nervous and Endocrine Physiology, Department of Biology, Faculty of Science, Kénitra, Morocco \\ Email: *yassine_chahirou@hotmail.fr, elhessni70@yahoo.com
}

How to cite this paper: Chahirou, Y., Lamtai, M., Mesfioui, A., Ouichou, A., Coulibaly, M., Boussekkour, R. and El Hessni, A. (2018) Impact of the Association of a High Fructose Diet and Chronic Mild Stress on Metabolic and Affective Disorders in Male Rat. Journal of Behavioral and Brain Science, 8, 157-170. https://doi.org/10.4236/jbbs.2018.84010

Received: February 17, 2018

Accepted: April 21, 2018

Published: April 24, 2018

Copyright $\odot 2018$ by authors and Scientific Research Publishing Inc. This work is licensed under the Creative Commons Attribution International License (CC BY 4.0).

http://creativecommons.org/licenses/by/4.0/

c) (i) Open Access

\begin{abstract}
An early unbalanced nutritional diet can induce affective disorders in adulthood. As well as stress in adolescence can accentuate these disorders. Both human and rat structural changes have been demonstrated in the hippocampus, likewise, oxidative stress may be involved in these disturbances. The objective of this study is to see the impact of a high-fructose diet (PN21) associated with chronic mild stress (CMS) at the end of adolescence (PN55) on metabolic and affective disorders in rats Wistar. This study was performed on four groups of male rats: control group, CMS for five weeks (PN55), fructose for ten weeks (PN21) and fructose for ten weeks (PN21) associated with CMS for five weeks (PN55). These animals underwent behavioral tests to evaluate their affective states (open field test, Sucrose preference test). After sacrifice, the dosage of glucose, triglycerides and total cholesterol was performed at the prefrontal cortex $(\mathrm{CPF})$ and also at the hippocampus; the dosage of nitric oxide (NO) was performed, too. The bulk of our results show that fructose induces metabolic disturbances; the CMS induces a state of depression-like, while the association potentiated metabolic disturbances, depression-like state and also inducing anxiety. This study has shown that fructose and CMS can disrupt the various functions of the body and their association can potentiate these disturbances.
\end{abstract}

\section{Keywords}

Fructose, CMS, Metabolic Disorders, Oxidative Stress, Anxiety, Depression

\section{Introduction}

Obesity is one of the major health problems in the world, and its prevalence continues to increase. This epidemic is accompanied by metabolic and behavior- 
al disorders [1] [2]. These diseases are due to genetic and epigenetic factors especially food [3]. Food is essential for the proper functioning of the body, however, an excess leads to harmful health effects, especially fructose, which has been heavily consumed during the last years [3]. The role of dietary fructose has raised a lot of worries about its close relationship with the development of metabolic diseases [4]. Fructose absorbed by the hepatic portal vein is almost completely metabolized in the liver via the action of fructokinase [5] which is an insulin-independent step, and therefore, fructose is metabolized largely in the absence of insulin secretion. Fructose is rapidly converted in the liver to phosphate triose independently of the action of insulin [6]. A quantity of phosphate triose can also form acetyl COA which is also converted to free fatty acids which will be re-esterified to triglycerides in liver cells by a process of lipogenesis [7], which appears to be associated with increased Plasma triglyceride (TG) and very low density lipoprotein (VLDL) concentrations with development of hepatic steatosis, glucose intolerance, insulin resistance and the development of hypertension [8] [9]. Another factor, whose intensity can be decisive for health, is stress, when experienced at an early age and/or chronically [10] [11]. It causes various disturbances within the body including those related to the nervous system: depression and anxiety. These mental disorders have adverse psychological, physical and socio-economic repercussions. They are responsible for a high mortality by suicide, to which is added the excess mortality non-suicidal (accidental, consumption of alcohol, tobacco or drugs) especially during the physiological and behavioral transition phase which is adolescence [12]. These factors have a negative impact on the patient and his entourage. Of this scourge, which is formed by psychiatric pathologies, major depression is the most prevalent disorder in the world, followed by anxiety, the prevalence of which still holds second place in primary health care facilities worldwide according to statistics of WHO. In addition, anxiety and depressive disorders combine in about half of all subjects [13]. The aim of this study is to evaluate the effects of a high-fructose diet at an early age (PN21), a CMS at the end of adolescence (PN55) and their association on the biochemical profile and affective disorders in Wistar rat in adulthood.

\section{Material and Methods}

\subsection{Animals}

Right after weaning, male Wistar rats (21D) and body weight $30 \pm 2 \mathrm{~g}$, born and reared at the laboratory pet shop in optimal conditions according to the faculty's ethics committee, were manipulated. These animals were born and raised in pet Shop at a temperature varying between $20^{\circ} \mathrm{C}-25^{\circ} \mathrm{C}$, distributed in scientific cages, lined with a litter made of wood chips which was renewed every two days, with light regulated automatically $(7 \mathrm{~h}-19 \mathrm{~h} \mathrm{ON})$. The animals are always handled in the same time intervals and by the same experimenter. The experimental protocol was performed in accordance with the guidelines of the OECD (Organization for Economic Co-operation and Development). 
The animals were divided into four groups:

1) Group exposed to a standard diet (PN21): (control).

2) Group exposed to a high fructose diet (23\%) (PN21): (Fructose).

3) Group exposed to a standard diet and a CMS during the end of adolescence (PN55): (Stress).

4) Group exposed to a high fructose diet (23\%) (PN21) and a CMS during the end of adolescence (PN55): (Fructose/stress).

\subsection{Diets}

The rats had free access to tap water for drinking and to a:

1) Standard diet provided by the company Alf Sahel of marketing of animal feed, Morocco. (13\% crude protein, $2 \%$ fat, $0.3 \%$ phosphorus, $9 \%$ mineral, $15 \%$ cellulose, $1 \%$ calcium, $500 \mathrm{IU}$ vitamin A, $75 \mathrm{IU}$ vitamin D, 1 IU of vitamin E.)

2) High-calorie diet high in fructose at $23 \%$ dissolved in water [14].

\subsection{Chronic Mild Stress Protocol (CMS)}

As there are different stress protocols in the literature [10] [11] [15], we opted for a CMS that involves putting animals under various stressors daily. The disturbances applied are of a low intensity and without food or dipsic deprivation. The interest of the protocol is the repetition of low and unpredictable events for animals. The procedure of stressing the rats selected for this work was based on a standardized protocol [16] [17] [18]. The animals were exposed for five weeks to two stressors per day in an unpredictable way, these factors are: $45^{\circ}$ cage tilt (24 h), wet litter during (24 h), cage vibration (5 min), change waking/sleeping cycle $(36 \mathrm{~h})$, forced swimming with cold water $4^{\circ}(5 \mathrm{~min})$, forced swimming with hot water $40^{\circ}$ (5 $\left.\mathrm{min}\right)$, strong lighting $(12 \mathrm{~h})$, tightening the tail $(5 \mathrm{~min})$.

\subsection{Behavioral Tests.}

To evaluate behavioral disorders related to high calorie diet and stress, animals in adulthood have undergone a battery of tests.

1) Open field (OFT).

The open field or open field test [19], is a white test, which measures one square meter surrounded by four $40 \mathrm{~cm}$ high walls and placed under strong lighting. It was divided into 25 squares $(20 \mathrm{~cm} \times 20 \mathrm{~cm})$, defined as 9 central and 16 peripherals. At the beginning of the test, which lasts $10 \mathrm{~min}$, the animal was placed in the center of the device and its behavior is filmed for later analysis. The device was cleaned after each pass, using $70 \%$ ethanol. The parameters measured in this study are: the number of central squares visited, the number of total squares visited and the time spent in the central zone. These parameters are generally used as parameters measuring locomotor activity and anxiety [20].

2) Sucrose preference test (Sucrose test: ST).

This test allows evaluating the sensations of pleasure in the animal. It involves the simultaneous presentation of two baby bottles to the animal, one with a $4 \%$ 
sucrose solution and the other with tap water. The consumption of sweet solution gives pleasure to an animal whose hedonic system is working properly. Conversely, in a "depressed" animal whose hedonic system is affected, the craving for sugar will be less important. The exposure of the animal to the sweet solution lasted 8 days, the first four days are to habituate the animals while the last four days are to measure the volume of sweet solution and tap water consumed by these animals [21]. To avoid the possible effects of the position of the bottle, it was changed during the exposure (the two bottles were alternated during the last two days). The animals were not deprived beforehand of water or food.

The consumption of water and the $4 \%$ sucrose solution was estimated by weighing the bottles and measuring the remaining water volumes in the bottles after exposure.

\subsection{Biochemical Dosage}

1) Determination of glucose, triglycerides and total cholesterol.

The biochemical analyzes performed in this work consisted in measuring glucose, triglycerides and cholesterol in order to explore the metabolic profile of the animals.

All these analyses were carried out by the use of marketed reagents imported from the company (Bio Systems S. A, Spain) which must follow the protocol set by the manufacturer.

2) Determination of nitric oxide.

The determination of the NO is carried out according to the method of Griess, It is an indirect dosage, it is the nitrites, products of degradation of NO which are dosed. This assay consists of a diazotization reaction in two steps: the nitrites form a diazonium salt with sulfanilic acid which is then coupled with an amine (N-naphthylethylenediamine) to give an azo dye that absorbs at $548 \mathrm{~nm}$. The assay is carried out in 96-well plate. This assay was carried out by the Griess reagent, the composition of which is as follows: (Griess solution $\mathrm{A}=0.1 \%$ of naphthylethylenediamine dichlorohydrate diluted in water + Griess solution $\mathrm{B}=$ $1 \%$ of sulfanilamide diluted in $\mathrm{H}_{3} \mathrm{PO}_{4}$ at $5 \%$ ). In this study the concentration of NO in the hippocampus.

3) Data Analysis.

Behavioral data and biochemical parameters were analyzed by two-way ANOVA using SPSS version 22. Post hoc comparisons were made using the $\mathrm{Tu}$ key's test. All data are expressed as the means \pm standard error of the means (S.E.M.). Differences were considered significant when $p<0.05\left(^{*}\right)$, very significant when $p<0.01\left(^{* *}\right)$ and highly significant when $p<0.001\left(^{* * *}\right)$.

\section{Results}

1) Effect of a high fructose diet, a CMS and their association on weight gain (Figure 1).

Statistical analyzes showed that the high fructose diet increased body weight 


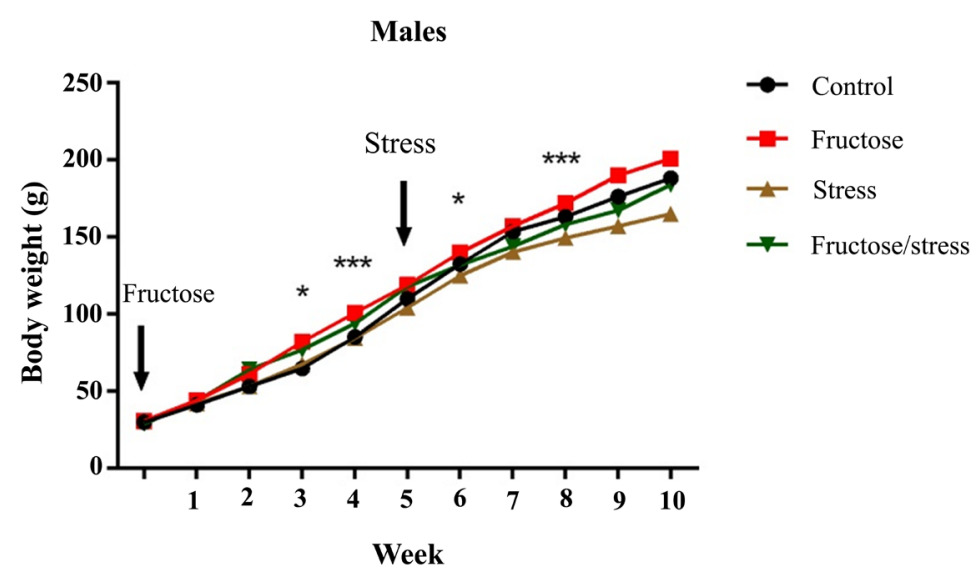

Figure 1. Effect of a high fructose diet, a CMS and their association on weight gain in rats. Results are expressed as mean \pm SEM. The significance level is 0.05 . ${ }^{*} p<0.05,{ }^{* *} p<$ $0.01,{ }^{* * *} p<0.001$.

significantly compared to the control group from the third week $(P=0.003)$. This difference became highly significant from the fourth week $(P<0.001)$. Complication by CMS during the fifth week induced changes in body weight: the CMS significantly decreased weight from the second week $(P=0.036)$ compared to the control group, this weight loss became highly significant from the third week $(P<0.001)$. The combination of high fructose diet and CMS induced a very significant weight loss compared to the Fructose group $(P=0.007)$, this fall became highly significant $(P<0.001)$ after four weeks of association.

2) Effect of a high fructose diet, a CMS and their association on the level of anxiety.

Level of anxiety measured in the OFT.

Statistical analyzes have shown that the high-fructose diet and the CMS have not induced any significant change in the number of center squares entred and time spent in center squares. While their association induced anxiety-like significantly decreasing the number of center squares entred $(P=0.018)$ (Figure 2(a)) and very significantly the time spent in center squares $(P=0.005)$ (Figure $2(b))$. This association does not induce any significant change of the parameter (number of total squares entred) (Figure 2(c)) which gives information on the locomotor activity of animals.

3) Effect of a high fructose diet, a CMS and their association on depressive behavior.

Depressive behavior measured in the sucrose preference test.

The sucrose-preference test revealed that the animals exposed to CMS reduced the consumption of sweet solution in a very significant way $(P=0.002)$, while the combination of CMS with the high-fructose diet decreased significantly this sucrose preference $(P=0.035)$ compared to the control group (Figure $3(\mathrm{a})$ ). In parallel, the animals exposed to the CMS increased the consumption of tap water in a very significant way compared to the control group $(P=0.002)$ and the Fructose group $(P=0.001)$ (Figure $3(\mathrm{~b}))$. 


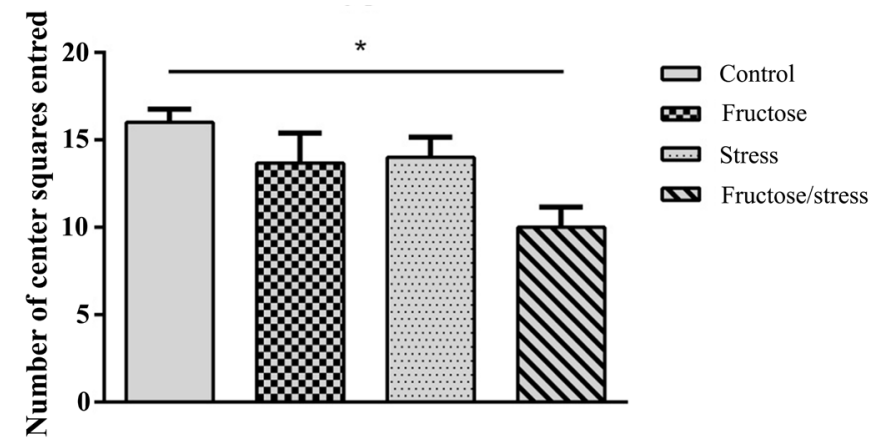

(a)
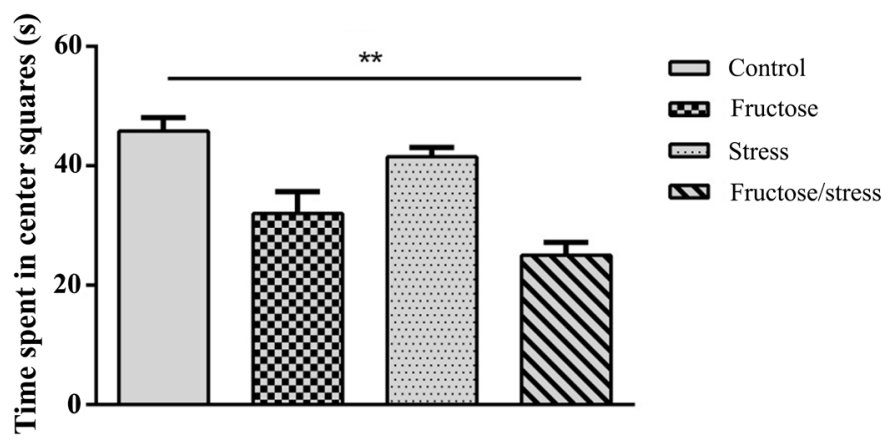

(b)

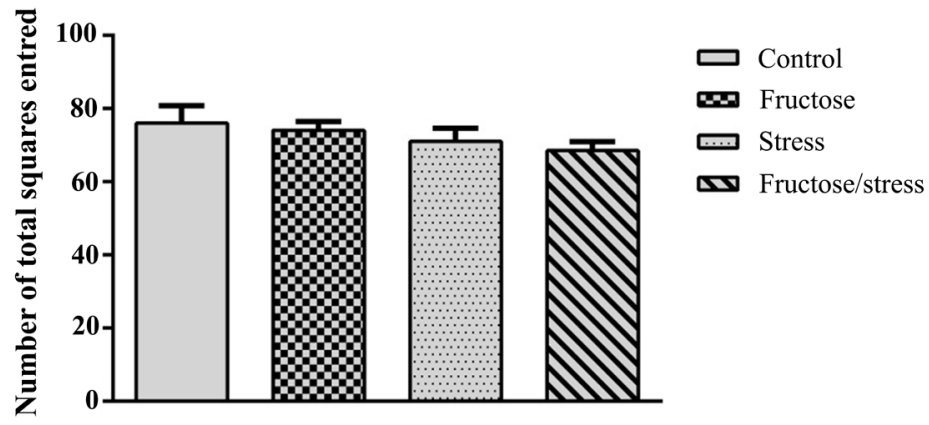

(c)

Figure 2. Effect of a high-fructose diet, a CMS and their association on the level of anxiety: (a) (Number of center squares entred); (b) (Time spent in center squares). (c) (Number of total squares entred). Results are expressed as mean \pm SEM. The significance level is $0.05 .{ }^{\star} p<0.05,{ }^{* *} p<0.01,{ }^{* *} p<0.001$.

4) Effect of a high fructose diet, a CMS and their association on the level of anxiety, level of glucose and level of triglyceride.

Statistical analyzes showed that the high fructose diet increased glycemia compared to the control group in a non-significant way. While the combination of a high fructose diet and the CMS significantly increased glycemia compared to control animals $(P=0.045)$ (Figure $4(a))$. The high fructose diet significantly increased triglyceridemia compared with control animals $(P=0.009)$, the complication of this diet by the CMS has the same effect $(P=0.004)$. This complication significantly increased the Triglyceridemia compared to the Stress group ( $P$ $=0.031$ ) (Figure 4(b)). Regarding the concentration of total cholesterol, the high 


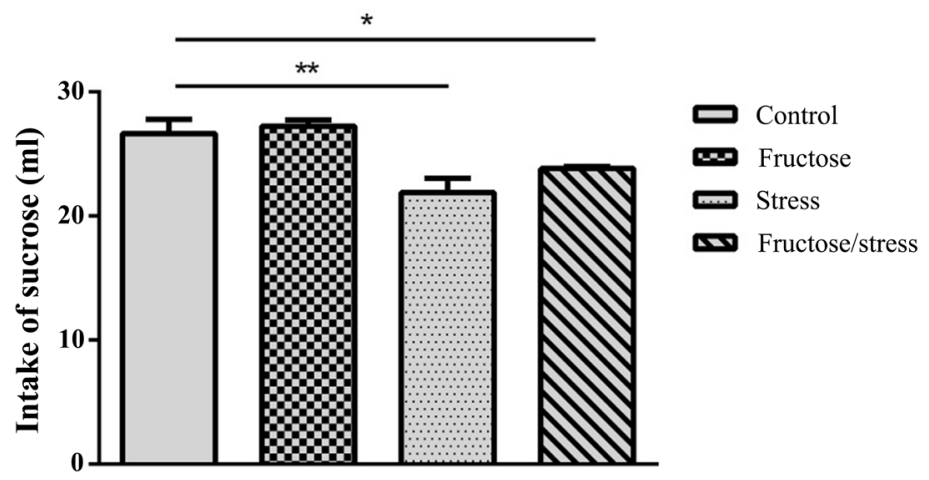

(a)

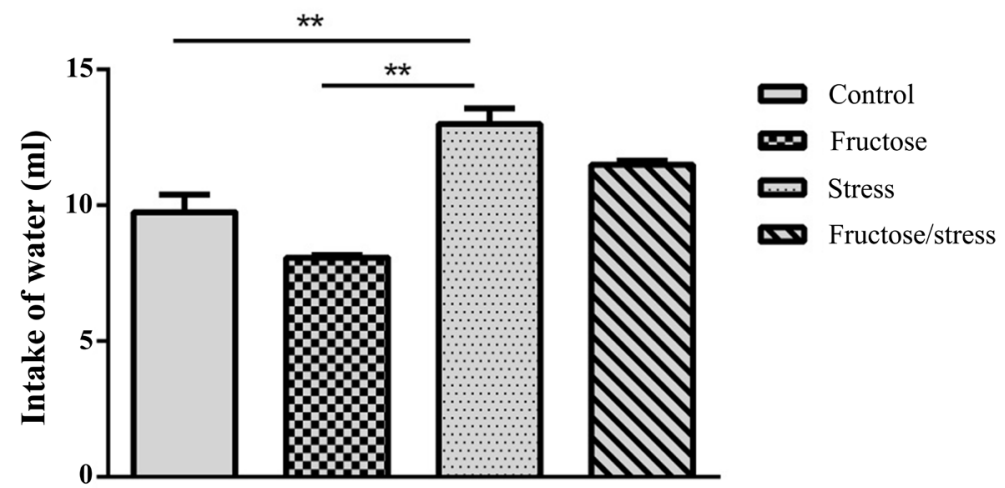

(b)

Figure 3. Effect of a high fructose diet, a CMS and their association on depressive behavior. (a) Intake of sucrose; (b) Intake of water. Results are expressed as mean $\pm \mathrm{SEM}$. The significance level is $0.05 .{ }^{\star} p<0.05,{ }^{* *} p<0.01,{ }^{* *} p<0.001$.

fructose diet and chronic stress did not significantly influence this concentration, whereas their combination increased the synthesis of total cholesterol very significantly compared to the control group $(P=0.002)$ and significantly compared to the Stress group $(P=0.02)$ (Figure $4(c)$ ).

5) Effect of a high fructose diet, a CMS and their association on oxidative stress.

Nitric oxide.

The measurement of nitric oxide (NO) in the hippocampus showed that the high fructose diet, the CMS and their association have no significant effect on NO concentration (Figure 5(a)). This association induced a very significant increase in NO in the prefrontal cortex $(P=0.001)$ (Figure $5(\mathrm{~b}))$.

\section{Discussion}

The purpose of this study is to evaluate the effects of combining a high fructose diet with chronic stress in late adolescence on biochemical and behavioral patterns. Our results show that high fructose diet does not induce any behavioral disorder. While the PN55-CMS induces anxiety and depressive disorder, these disorders are also observed in several studies [22] [23] [24]. The level of anxiety in a CMS model is generally due to activation of the corticotropic axis, which leads to the release of glucocorticoid hormones (GCs) and CRF [25] [26]. During 


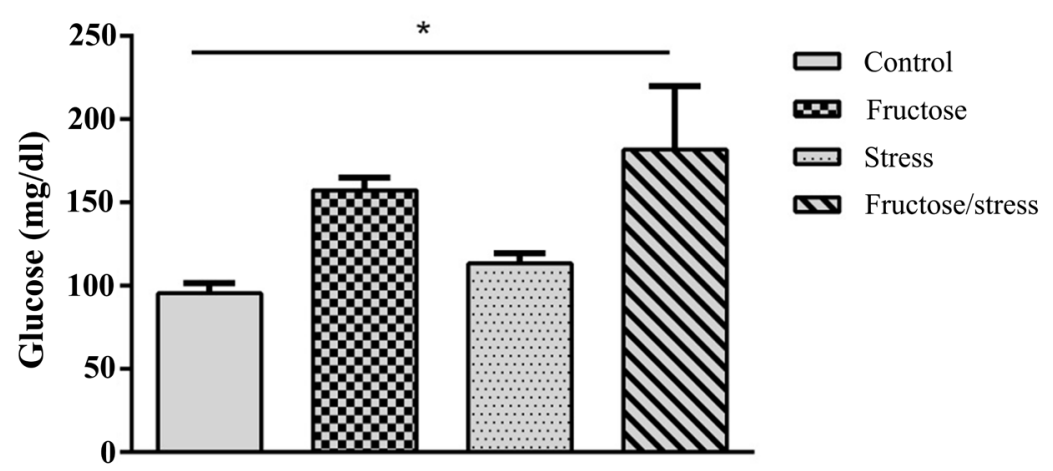

(a)

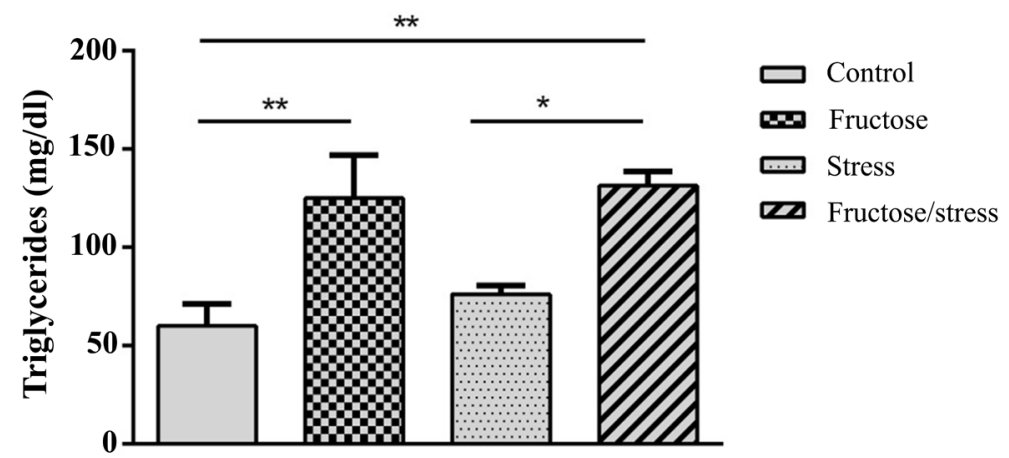

(b)

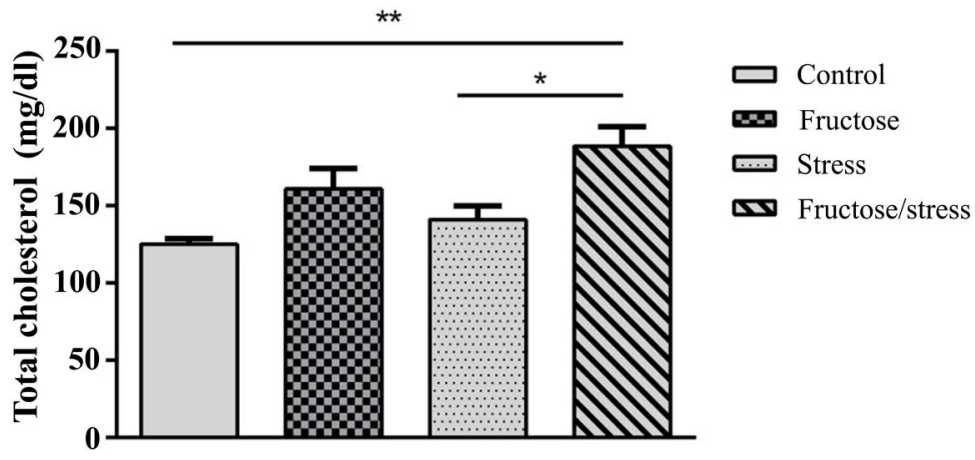

(c)

Figure 4. Effect of a high fructose diet, a CMS and their association on the biochemical profile (a): Glucose; (b): Triglycerides; (c): Total cholesterol. Results are expressed as mean \pm SEM. The significance level is $0.05 .{ }^{\star} p<0.05,{ }^{\star *} p<0.01,{ }^{\star * *} p<0.001$.

stress, the emotional or physical information is integrated by the appropriate structures (amygdala, hippocampus and PFC) and can directly activate the locus coerulus, generating a massive and very fast release of norepinephrine at the central level. This structure receives nerve fibers from the CRF neurons of PVN of the hypothalamus, central amygdala, and innervates the PVN with noradrenergic nerve fibers [27]. Following exposure to CMS or the combination of a high-fructose diet and CMS, depressive behavior is well established as confirmed by the decrease in sucrose preference, these results are in accordance with current literature [28] [29]. The major system that responds to stress is the corticotropic axis. The essential role of this system in the effects of chronic stress was demonstrated by experiments showing that the development of a depressive 


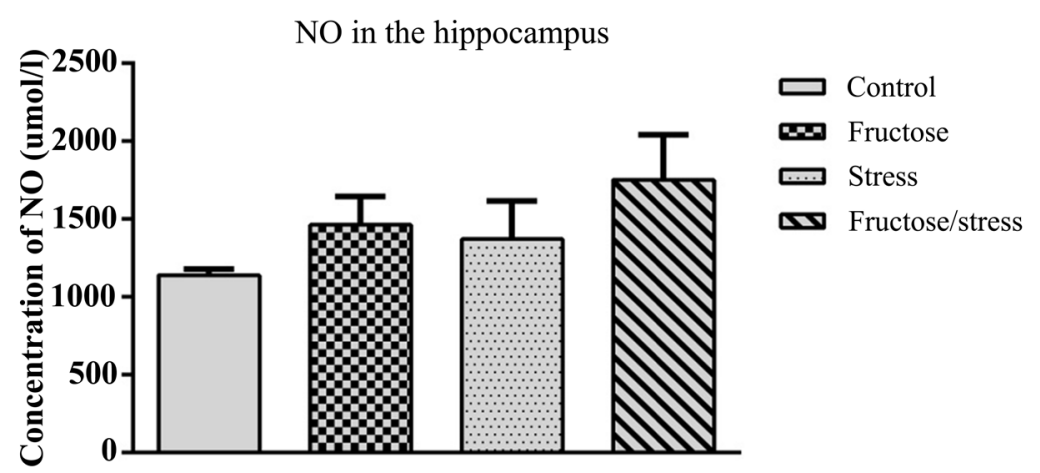

(a)

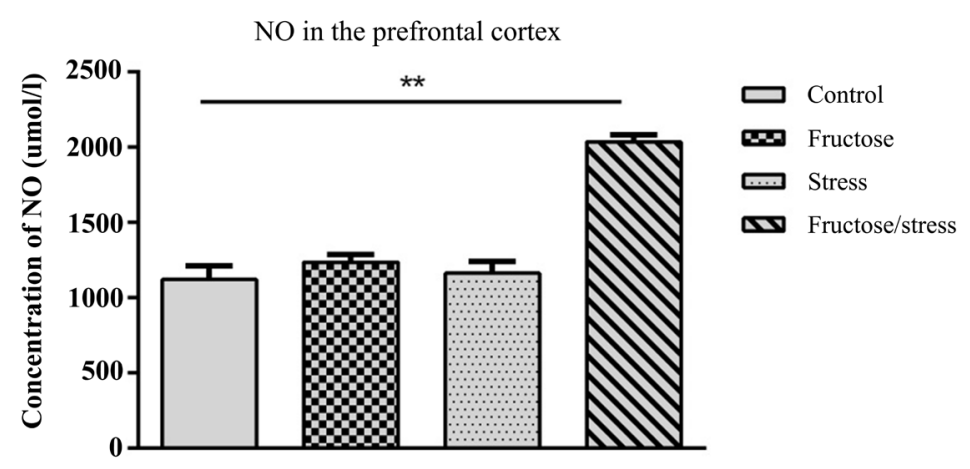

(b)

Figure 5. Effect of a high fructose diet, a CMS and their association on oxidative stress. (a): concentration of NO in the hippocampus; (b): concentration of NO in the prefrontal cortex. Results are expressed as mean \pm SEM. The significance level is $0.05 .{ }^{\star} p<0.05,{ }^{* *} p$ $<0.01,{ }^{* *} p<0.001$.

phenotype upon exposure to a CMS (decreased sucrose preference) is decreased by the glucocorticoid receptor antagonist (mifepristone) [30] or by the corticosterone synthesis inhibitor (metyrapone) [31] or by ablation of adrenal gland [32] [33]. Stress intensively stimulates the amygdala that projects towards the ventral pallidum inducing a decrease in dopamine discharge at the level of the nucleus accumbens (Nacc) by the ventral tegmental area (VTA) [34]. Thus, the activation of the lateral habenula nucleus, which is very well known in the management of negative emotions (aversion) via its projections towards the VTA and the raphe nucleus, consists respectively in the decrease of dopamine and 5HT production in the mesocorticolimbic pathway, this decreases the preference for sucrose [35]. Exposure to stress during adolescence is associated with structural and molecular changes [36]. Indeed, preclinical studies have shown that stress leads to a 5HT decrease in the amygdala and Nacc with a decrease in dopamine at PFC, HPC and Nacc. This molecular perturbation is at the origin of the morphological changes of the neurons [37]. Other studies have shown that increasing GC rates can affect the connections between structures involved in stress, which predispose to psychiatric pathologies [38]. These studies have shown that high levels of GCs reduce neurogenesis and synaptogenesis at the PFC and hip- 
pocampus [38]. Thus, chronic stress decreases metabolism and neurotrophic factors in the pyramidal cells of the median CPF: chronic exposure to glucocorticoids attenuates synaptic proteins (PSD95) and synapsin I [33] following a decrease in the production of BDNF at the median PFC and the hippocampus which causes a depressive state [39]. The PN21 hypercaloric diet and the PN55 CMS induce a non-significant increase in the concentration of glucose and total cholesterol. While their association has potentiated metabolic disorders: increased blood sugar, triglyceride and total cholesterol. Many studies have shown that fructose has deleterious effects on glucose homeostasis and insulin sensitivity [40] [41]. Our results show that chronic stress has greatly potentiated the deleterious effect of fructose, which causes metabolic disorders whose incidence continues to increase, leading to increased risks of diabetes and cardiovascular diseases. Hepatic discoloration revealed by macroscopic observation is a sign of the accumulation of fat, which causes hepatic steatosis, this condition is often associated with insulin resistance. Stress by activating the sympathetic nervous system and blocking the production of insulin, aggravates the deleterious effects caused by fructose, lipid metabolism is also altered by stress [42]. This combination induced an increase in NO [43]. This free radical has a very high capacity to activate intracellular proteins such as (NFkB, PKC, MAPK) that participate in the establishment of cellular damage at the peripheral and central level. Free radicals and inflammation are at the origin of metabolic diseases and are also the pathophysiological cause of affective disorders. The work of Barone and these collaborators in 2016 [44] showed that a state of insulin resistance is accompanied by psychiatric and neurodegenerative diseases. The same studies have shown that a diet rich in fat and fructose increased leptinemia and insulinemia, as well as the rate of TNF $\alpha$, IL6, IL1 at the level of the hippocampus [45]. These pro-inflammatory brain cytokines (in particular IL-1, IL- 6 and TNF $\alpha$ ) will act on neural and non-neural cells through their specific receptors and thus contribute to the development of neuropsychiatric disorders. The mechanisms by which cytokines induce depressive states have been studied in particular in patients treated with immunotherapy (interferon- $\gamma$ for example). These studies show that cytokines influence the development of depression by acting on the metabolism of monoamines. Interferon- $\gamma$ or TNF- $\alpha$ activates the enzyme IDO (indolamine 2, 3 dioxygenase) which converts tryptophan into kynurenine. However, tryptophan is also the precursor of serotonin which is decreased by the activation of the IDO in the PFC and in the hippocampus. Cytokines also have an effect on the bioavailability of brain dopamine leading to psychomotor retardation and anhedonia. This study has shown that the combination of a PN21 high fructose diet and the PN55-CMS induces metabolic and effective disorders in adulthood.

\section{References}

[1] Viesselman, J.O. and Roig, M. (1985) Depression and Suicidality in Eating Disord- 
ers. Journal of Clinical Psychiatry, 46, 118-124.

[2] Casper, R.C. (1998) Depression and Eating Disorders. Depress Anxiety, 8, 96-104.

[3] Wolf, A., Bray, G.A. and Popkin, B.M. (2008) A Short History of Beverages and How Our Body Treats Them. Obesity Reviews, 9, 151-164. https://doi.org/10.1111/j.1467-789X.2007.00389.x

[4] Misra, A. and Khurana, L. (2009) Obesity and the Metabolic Syndrome in Developing Countries. Journal of Clinical Endocrinology \& Metabolism, 93, 9-30. https://doi.org/10.1210/jc.2008-1595

[5] Heinz, F., Lamprecht, W. and Kirsch, J. (1968) Enzymes of Fructose Metabolism in Human Liver. Journal of Clinical Investigation, 47, 1826-1832. https://doi.org/10.1172/JCI105872

[6] Mayes, P.A. (1993) Intermediary Metabolism of Fructose. American Journal of Clinical Nutrition, 58, 754-765. https://doi.org/10.1093/ajcn/58.5.754S

[7] Parks, E.J., Skokan, L.E., Timlin, M.T. and Dingfelder, C.S. (2008) Dietary Sugars Stimulate Fatty Acid Synthesis in Adults. Journal of Nutrition, 138, 1039-1046. https://doi.org/10.1093/jn/138.6.1039

[8] Le KA, T.L. (2007) Metabolic Effects of Fructose. Curr Opin Clin Nutr Metabol Care, 10, 210-214.

[9] Havel, P.J. (2005) Dietary Fructose: Implication for Dysregulation of Energy Homeostasis and Lipid/Carbohydrate Metabolism. Nutrition Reviews, 63, 133-137. https://doi.org/10.1111/j.1753-4887.2005.tb00132.x

[10] Strekalova, T. and Steinbusch, H.W. (2010) Measuring Behavior in Mice with Chronicstress Depression Paradigm. Progress in Neuro-Psychopharmacology and Biological Psychiatry, 34, 348-361. https://doi.org/10.1016/j.pnpbp.2009.12.014

[11] Xue, X., Shao, S., Li, M., Shao, F. and Wang, W. (2013) Maternal Separation Induces Alterations of Serotonergic System in Different Aged Rats. Brain Research Bulletin, 95, 15-20. https://doi.org/10.1016/j.brainresbull.2013.03.003

[12] Rouillon, F. (2008) Epidémiologie des troubles psychiatriques. Annales Médico-Psychologiques, revue psychiatrique, 166, 63-70.

https://doi.org/10.1016/j.amp.2007.11.010

[13] Zimmerman, M., McDermut, W. and Mattia, J.I. (2000) Frequency of Anxiety Disorders in Psychiatric Outpatients with Major Depressive Disorder. American Journal of Psychiatry, 157, 1337-1340. https://doi.org/10.1176/appi.ajp.157.8.1337

[14] Lindqvist, A., Baelemans, A. and Erlanson-Albertsson, C. (2008) Effects of Sucrose, Glucose and Fructose on Peripheral and Central Appetite Signals. Regulatory Peptides, 150, 26-32. https://doi.org/10.1016/j.regpep.2008.06.008

[15] Strekalova, T., Couch, Y., Kholod, N., Boyks, M., Malin, D., Leprince, P., et al. (2011) Update in the Methodology of the Chronic Stress Paradigm: Internal Control Matters. Behavioral and Brain Functions, 7, 1-18. https://doi.org/10.1186/1744-9081-7-9

[16] Willner, P. (2005) Chronic Mild Stress (CMS) Revisited: Consistency and Behavioural Neurobiological Concordance in the Effects of CMS. Neuropsychobiology, 52, 90-110. https://doi.org/10.1159/000087097

[17] Li, N., Liu, R.J., Dwyer, J.M., Banasr, M., Lee, B., Son, H., et al. (2011) Glutamate N-methyl-D-aspartate Receptor Antagonists Rapidly Reverse Behavioral and Synaptic Deficits Caused by Chronic Stress Exposure. Biological Psychiatry, 69, 754-761. https://doi.org/10.1016/j.biopsych.2010.12.015

[18] Willner, P. (1997) The Chronic Mild Stress Procedure as an Animal Model of De- 
pression: Valid, Reasonably Reliable, and Useful. Psychopharmacology, 134, 371-377. https://doi.org/10.1007/s002130050473

[19] Archer, J. (1973) Tests for Emotionality in Rats and Mice: A Review. Animal Behaviour, 21, 205-235. https://doi.org/10.1016/S0003-3472(73)80065-X

[20] Strekalova, T., Spanagel, R., Dolgov, O. and Bartsch, D. (2005) Stress-Induced Hyperlocomotion as a Confounding Factor in Anxiety and Depression Models in Mice. Behavioural Pharmacology, 16, 171-180. https://doi.org/10.1097/00008877-200505000-00006

[21] Eagle, A.L., Mazei-Robison, M. and Robison, A.J. (2016) Sucrose Preference Test to Measure Stress-Induced Anhedonia. http://www.bio-protocol.org/e1822

[22] Zhu, W.L., Shi, H.S., Wang, S.J., Wu, P., Ding, Z.B. and Lu, L. (2011) Hippocampal CA3 Calcineurin Activity Participates in Depressive-Like Behavior in Rats. Journal of Neurochemistry, 117, 1075-1086. https://doi.org/10.1111/j.1471-4159.2011.07285.x

[23] Chiba, S., Numakawa, T., Ninomiya, M., Richards, M.C., Wakabayashi, C. and Kunugi, H. (2012) Chronic Restraint Stress Causes Anxiety- and Depression-Like Behaviors, Downregulates Glucocorticoid Receptor Expression, and Attenuates Glutamate Release Induced by Brain-Derived Neurotrophic Factor in the Prefrontal Cortex. Progress in Neuro-Psychopharmacology \& Biological Psychiatry, 39, 112-119. https://doi.org/10.1016/j.pnpbp.2012.05.018

[24] Eiland, L., Ramroop, J., Hill, M.N., Manley, J. and McEwen, B.S. (2012) Chronic Juvenile Stress Produces Corticolimbic Dendritic Architectural Remodeling and Modulates Emotional Behavior in Male and Female Rats. Psychoneuroendocrinology, 37, 39-47. https://doi.org/10.1016/j.psyneuen.2011.04.015

[25] Matta, S.G., Linner, K.M. and Sharp, B.M. (1993) Interleukin-1 Alpha and Interleukin-1 Beta Stimulate Adrenocorticotropin Secretion in the Rat through a Similar Hypothalamic Receptor(s): Effects of Interleukin-1 Receptor Antagonist Protein. Neuroendocrinology, 57, 14-22. https://doi.org/10.1159/000126336

[26] Korte, S.M. (2001) Corticosteroids in Relation to Fear, Anxiety and Psychopathology. Neuroscience and Biobehavioral Reviews, 25, 117-142. https://doi.org/10.1016/S0149-7634(01)00002-1

[27] Dunn, A.J. and Swiergiel, A.H. (2008) The Role of Corticotropin-Releasing Factor and Noradrenaline in Stress-Related Responses, and the Inter-Relationships between the Two Systems. European Journal of Pharmacology, 583, 186-193. https://doi.org/10.1016/j.ejphar.2007.11.069

[28] Nesher, E., Gross, M., Lisson, S., Tikhonov, T., Yadid, G. and Pinhasov, A. (2013) Differential Responses to Distinct Psychotropic Agents of Selectively Bred Dominant and Submissive Animals. Behavioural Brain Research, 236, 225-235. https://doi.org/10.1016/j.bbr.2012.08.040

[29] Moussaieff, A., Gross, M., Nesher, E., Tikhonov, T., Yadid, G. and Pinhasov, A. (2012) Incensole Acetate Reduces Depressive-Like Behavior and Modulateshippocampal BDNF and CRF Expression of Submissive Animals. Journal of Psychopharmacology, 26, 1584-1593. https://doi.org/10.1177/0269881112458729

[30] Wu, L.M., Han, H., Wang, Q.N., Hou, H.L., Tong, H., Yan, X.B. and Zhou, J.N. (2007) Mifepristone Repairs Region-Dependent Alteration of Synapsin I in Hippocampus in Rat Model of Depression. Neuropsychopharmacology, 32, 2500-2510.

[31] Kvarta, M.D., Bradbrook, K.E., Dantrassy, H.M., Bailey, A.M. and Thompson, S.M. (2015) Corticosterone Mediates the Synaptic and Behavioral Effects of Chronic 
Stress at Rat Hippocampal Temporoammonic Synapses. Journal of Neurophysiology, $114,1713-1724$

[32] Goshen, I., Kreisel, T., Ben-Menachem-Zidon, O., Licht, T., Weidenfeld, J., Ben-Hur, T. and Yirmiya, R. (2008) Brain Interleukin-1 Mediates Chronic Stress-Induced Depression in Mice via Adrenocortical Activation and Hippocampal Neurogenesis Suppression. Molecular Psychiatry, 13, 717-728.

[33] Chen, J., Wang, Z.Z., Zuo, W., Zhang, S., Chu, S.F. and Chen, N.H. (2016) Effects of Chronic Mild Stress on Behavioral and Neurobiological Parameters-Role of Glucocorticoid. Hormones and Behavior, 78, 150-159.

[34] Chang, C.H. and Grace, A.A. (2014) Amygdala-Ventral Pallidum Pathway Decreases Dopamine Activity after Chronic Mild Stress in Rats. Biological Psychiatry, 76, 223-230.

[35] Willner, P. (2016) Reliability of the Chronic Mild Stress Model of Depression: A User Survey. Neurobiology of Stress, 6, 68-77.

[36] Hunter, R.G., McCarthy, K.J., Milne, T.A., Pfaff, D.W. and McEwen, B.S. (2009) Regulation of Hippocampal H3 Histone Methylation by Acute and Chronic Stress. Proceedings of the National Academy of Sciences, 106, 20912-20917. https://doi.org/10.1073/pnas.0911143106

[37] Dannlowski, U., et al. (2008) 5-HTTLPR Biases Amygdala Activity in Response to Masked Facial Expressions in Major Depression. Neuropsychopharmacology, 33, 418-424. https://doi.org/10.1038/sj.npp.1301411

[38] Wei, Q., et al. (2004) Glucocorticoid Receptor Overexpression in Forebrain: A Mouse Model of Increased Emotional Lability. Proceedings of the National Academy of Sciences, 101, 11851-11856. https://doi.org/10.1073/pnas.0402208101

[39] Filho, C.B., Jesse, C.R., Donato, F., Giacomeli, R., Del Fabbro, L., da Silva Antunes, M., de Gomes, M.G., Goes, A.T., Boeira, S.P., Prigol, M. and Souza, L.C. (2015) Chronic Unpredictable Mild Stress Decreases BDNF and NGF Levels and $\mathrm{Na}(\mathrm{p})$, $\mathrm{K}(\mathrm{p})$-ATPase Activity in the Hippocampus and Prefrontal Cortex of Mice: Antidepressant Effect of Chrysin. Neuroscience, 289, 367-380.

[40] Yolanda, B., Lombardo, A.E., Gustavo Hein, A.E. and Adriana, C. (2006) Metabolic Syndrome: Effects of n-3 PUFAs on a Model of Dyslipidemia, Insulin Resistance and Adiposity. Lipids, 42, 427-437.

[41] Robbez-Masson, V., Lucas, A., Gueugneau, A.M., Macaire, J.P., Paul, J.P., Grynberg, A. and Rousseau, D. (2008) Long-Chain (n-3) Polyunsaturated Fatty Acids Prevent Metabolic and Vascular Disorders in Fructose-Fed Rats. The Journal of Nutrition, 138, 1915-1922. https://doi.org/10.1093/jn/138.10.1915

[42] Catalina-Romero, C., Calvo, E., Sanchez-Chaparro, M.A., Valdivielso, P., Sainz, J.C., Cabrera, M., Gonzalez-Quintela, A. and Roman, J. (2013) The Relationship between Job Stress and Dyslipidemia. Scandinavian Journal of Public Health, 41, 142-149. https://doi.org/10.1177/1403494812470400

[43] Saad, A., Virella, G., Chassereau, Ch., et al. (2006) OxLDL Immune Complexes Activate Complement and Induce Cytokine Production by MonoMac 6 Cells and Human Macrophages. The Journal of Lipid Research, 47, 1975-1983. https://doi.org/10.1194/jlr.M600064-JLR200

[44] Barone, E., Di Domenico, F., Cassano, T., Arena, A., Tramutola, A., Lavecchia, M.A., Coccia, R., Butterfield, D.A. and Perluigi, M. (2016) Impairment of Biliverdin Reductase-A Promotes Brain Insulin Resistance in Alzheimer Disease: A New Paradigm. Free Radical Biology \& Medicine, 91, 127-142.

https://doi.org/10.1016/j.freeradbiomed.2015.12.012 
[45] De Sousa Rodrigues, M.E., Bekhbat, M., Houser, M.C., Chang, J., Walker, D.I., Jones, D.P., Oller do Nascimento, C.M.P., Barnum, C.J. and Tansey, M.G. (2016) Chronic Psychological Stress and High-Fat Highfructose Diet Disrupt Metabolic and Inflammatory Gene Networks in the Brain, Liver, and Gut and Promote Behavioral Deficits in Mice. Brain, Behavior, and Immunity, 59, 158-172. 